\title{
ULTRA-HIGH ASPECT-RATIO PDMS MICROPILLARS WITH SELF-ALIGNED MICROSPHERES FOR AIR-FLOW SENSING
}

\author{
Jungwook Paek and Jaeyoun Kim
}

Department of Electrical and Computer Engineering, Iowa State University, Ames, Iowa, USA

\begin{abstract}
We developed a new technique that enables routine fabrications of high aspect-ratio PDMS micropillars. The key enabling factor is the adoption of the direct drawing technique incorporated with the in situ heating for simultaneous hardening and solidification of the PDMS micropillars. In addition, our technique allows self-aligned installation of highly reflective microspheres at the tips of the micropillars. Using the transparent PDMS micropillar as a flexible waveguide and the microsphere as a self-aligned reflector, we transformed the microsphere-tipped PDMS micropillars into all optically interrogated air-flow sensors and successfully demonstrated the air-flow sensing capability.
\end{abstract}

\section{INTRODUCTION}

Micropillars made of PDMS have seen rapid expansion in their application scope, from bio-mimetic dry adhesion [1] and surface wetting control [2] to cell mechanics studies [3]. The newest addition to their application list is mass flow transduction where PDMS micropillars with higher aspect ratios are preferred for their greater deformability, enabling highly sensitive transduction in a small form factor. Realizing high aspect ratio PDMS micropillars, however, is a difficult challenge, especially with the use of conventional, replica-molding-based fabrication techniques [4]. The main obstacle is the need to de-mold the PDMS micropillars after curing. Despite the efforts to improve the yield of the process [5], the failure rate increases inevitably with the aspect-ratio. Another source of difficulty is the inherently low Young's modulus, $E_{\mathrm{Y}}$ of PDMS (typically $\sim 1 \mathrm{MPa}$ ) [6], which frequently leads to the collapse of the micropillar and then irreversible bonding to the substrate [7].

In this report, we present a new technique that enables routine fabrications of PDMS micropillars with aspect ratios higher than 40 [8]. The key enabling factor is the adoption of the direct drawing technique incorporated with the in situ thermal hardening for the realization of the high aspect-ratio PDMS micropillars, eliminating the de-molding process. To date, the direct drawing technique has been applied to polystyrene [9], PMMA [10], PTT [11], nylon [12], sucrose [13], polyurethane [14], SU-8 [15], and adhesives [16]. Its application to PDMS, however, has not been reported due mainly to the material's softness and slow solidification rate. We address the issues by incorporating in situ heating into the drawing process so that the micropillar gets simultaneously hardened and solidified during its formation. In addition, our technique allows self-aligned installation of highly reflective microspheres at the tips of the micropillars, leading to the realization of microsphere-tipped micropillars. Using the transparent PDMS micropillar as a flexible waveguide and the microsphere as a self-aligned reflector, we transformed the microsphere-tipped PDMS micropillars into all optically interrogated air-flow sensors and successfully demonstrated the air-flow sensing capability.

\section{FABICATION OF PDMS MICROPILLARS}

The PDMS MSMPs (microsphere-tipped micropillars) fabrication steps are depicted in Figure 1. We started out with the preparation of the drawing probe array. The microspheres were first assembled on a photo-lithographically patterned Su- 8 grid and then transferred onto a piece of double-stick tape attached to a glass substrate shown in Figure 1a. We used two types of microspheres as the drawing probes: $\sim 50 \mu \mathrm{m}$-diameter PMMA microspheres and $\sim 58 \mu \mathrm{m}$-diameter Ag-coated hollow glass microspheres. The latter was more effective for increasing the levels of reflection and tip-visibility. We then flipped the microsphere array and attached it to a micro-manipulator. Also, a $25 \mu \mathrm{m}$ PDMS layer was prepared and then pre-baked at $100^{\circ} \mathrm{C}$ on a hot plate. During the pre-bake, we lowered the microsphere array towards the PDMS thin film until they got separated by $50 \mu \mathrm{m}$. Upon completion of the pre-bake, we further lowered the microsphere array so that it can physically contact the PDMS thin film, as shown in Figure 1c. After $40 \mathrm{sec}$, we lifted the micro-manipulator, drawing PDMS micropillars as shown in Figure 1d. The temperature was maintained at $100^{\circ} \mathrm{C}$ throughout these steps for simultaneous solidification and hardening of the PDMS micropillars. Completed PDMS micropillars were then post-baked at $100^{\circ} \mathrm{C}$ for 2 hours and at room-temperature for 24 hours. After the post-bake, we further lifted the micro-manipulator so that the micropillars got elongated by $100 \%$. The resulting tension induces gradual detachment of the microspheres from the double stick tape, leading to the realization of PDMS MSMPs as shown in Figure 1e.
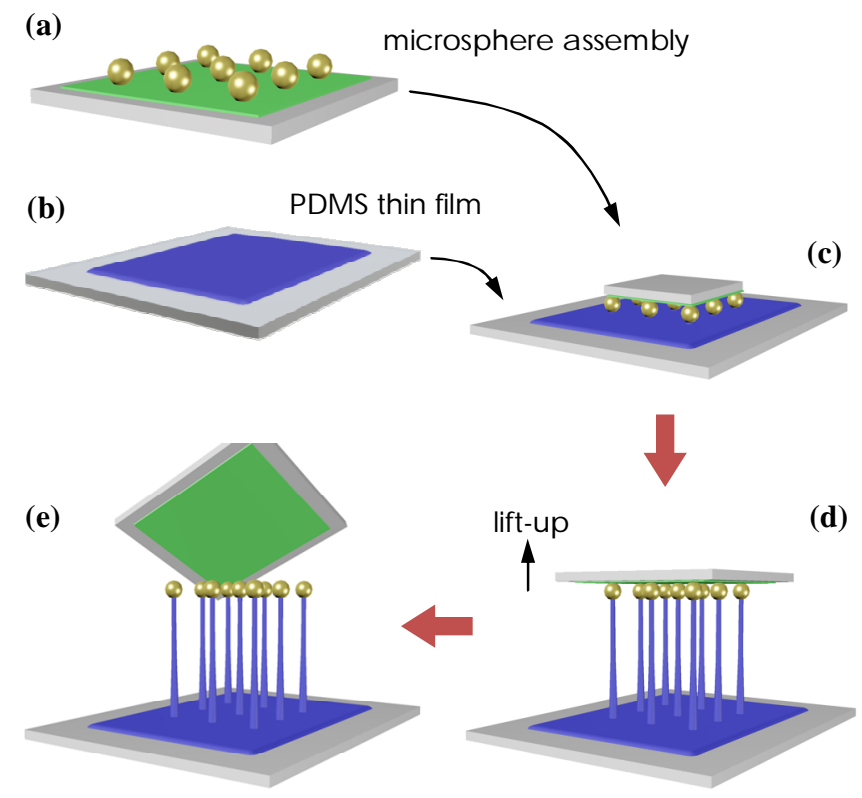

Figure 1. Fabrication flow (a) Microsphere assembly (b) PDMS thin film (c) After pre-baking of the PDMS thin film, the microsphere array physically contacted the PDMS thin film followed by an additional baking. (d) Directly drawing by lifting the microsphere array and the completed micropillars were then post-baked. (e) The double-stick tape was manually detached from the microspheres, completing the realization of the MSMPs 
At this step, the main cause of failure is insufficient curing of the PDMS micropillar which detaches the microsphere from the micropillar, rather than from the tape.

\section{PHYSICAL CHARACTERISTICS OF PDMS MICROPILLARS}

The direct drawing technique enabled routine fabrications of mm-scale PDMS micropillars with aspect-ratios > 40. Here, we defined the aspect-ratio as the ratio between $h$, the height and $d_{\text {ave }}$, the average of the top, middle and base diameters of the PDMS micropillar. The micropillar in Figure 2a was drawn with a 50 $\mu \mathrm{m}$-diameter PMMA microsphere. With its $h$ and $d_{\text {ave }}$ at 1134 and $13.5 \mu \mathrm{m}$, respectively, the micropillar exhibits an aspect-ratio of 84 . Such a high aspect-ratio was attainable for arrayed micropillars as well. The micropillars in the $4 \times 4$ array shown in Figure $2 b$ were drawn with a matching array of $58 \mu$ m-diameter Ag-coated hollow glass microspheres. In average, they were $877.2 \mu \mathrm{m}$ high and tapered with their diameter changing from $14.4 \mu \mathrm{m}$ right underneath the microsphere to $29.8 \mu \mathrm{m}$ at the base. With $20.8 \mu \mathrm{m}$ in $d_{\text {ave }}$, their average aspect-ratio was 42 . As pointed out above, capping the tip with a metallic microsphere greatly enhanced the micropillar's tip-visibility. Figure $2 \mathrm{c}$ shows the highest micropillar obtained so far. With its $h$ and $d_{\text {ave }}$ at 2400 and $21.3 \mu \mathrm{m}$, respectively, the aspect-ratio reached 112 . To the best of our knowledge, this is the highest aspect-ratio reported for a vertical PDMS micropillar. Figure $2 \mathrm{~d}$ shows the relation between $h$ and $d_{\mathrm{ave}}$ of micropillars. Initially, the aspect-ratio increases with $h$. However, beyond $h \sim$ $1.1 \mathrm{~mm}$, the increase in $h$ induces a matching increase in the base diameter, saturating the aspect-ratio in the range of $70 \sim 110$.

From the observations that the directly drawn PDMS micropillars can stand upright and support the weight of microspheres despite their thinness and high aspect-ratio, we conjectured that the in situ thermal hardening has indeed hardened PDMS. To quantify the impact of the in situ heating on PDMS hardening, we measured the PDMS micropillar's Young's modulus, $E_{\mathrm{Y}}$ based on the reference cantilever method [17] as shown in the inset of Figure $2 \mathrm{e}$, in which a reference cantilever with known spring constant $K_{r}$ is used to deflect the test cantilever and its spring constant $K_{t}$ is estimated from the relation $K_{t} \cdot \delta_{t}=K_{r} \cdot \delta_{r}$, where $\delta_{\mathrm{t}}$ and $\delta_{\mathrm{r}}$ represent the deflection lengths of the test and reference cantilevers, respectively. For conically tapered beams, $K$ and $E_{\mathrm{Y}}$ are related by $K=3 \pi \cdot E_{\mathrm{Y}} \cdot d^{4} \cdot T /\left(64 \cdot h^{3}\right)$ where $T$ is the taper constant defined as $d_{\min } / d_{\max }$ [18]. We chose a section of commercial PMMA fiber $\left(E_{\mathrm{Y}} \sim 3.0 \mathrm{GPa}\right)$ as the reference cantilever and the two MSMPs in Table 1 were targeted for this characterization. From the results shown in Figure 2e, $E_{\mathrm{Y}}$ of MSMP-A and MSMP-B was calculated as $2.45 \mathrm{MPa}$ and $2.31 \mathrm{MPa}$, respectively, which are higher than most $E_{\mathrm{Y}}$ values for bulk PDMS ( $\left.1 \mathrm{MPa}\right)$. Therefore, we attribute the $\sim 1$ $\mathrm{MPa}$ increase in $E_{\mathrm{Y}}$ to the in situ thermal hardening incorporated into the direct drawing process so that the directly drawn microsphere-tipped PDMS micropillars can stand upright despite of their thinness and high aspect-ratio.

Table 1: Characteristics of the PDMS micropillars under test

\begin{tabular}{|c|c|c|c|}
\hline & $h[\mu \mathrm{m}]$ & $d_{\text {ave }}[\mu \mathrm{m}]$ & Aspect Ratio \\
\hline MSMP-A & 800 & 19.8 & 40.4 \\
\hline MSMP-B & 1600 & 22 & 72.7 \\
\hline
\end{tabular}

(a)

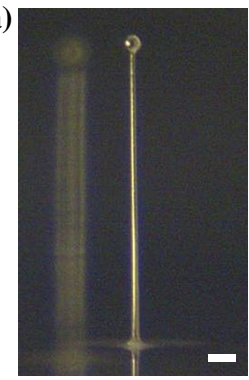

(b)

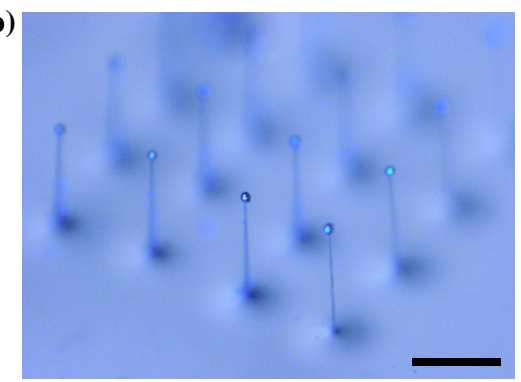

(c)

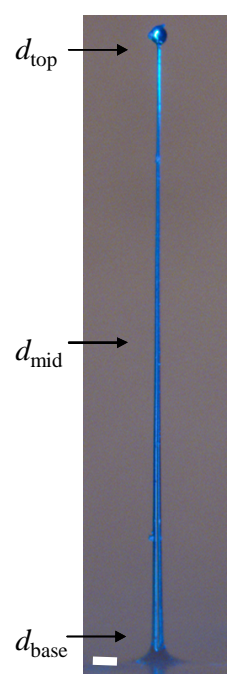

(d)

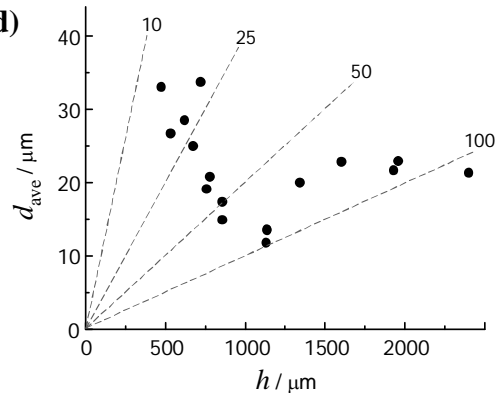

(e)

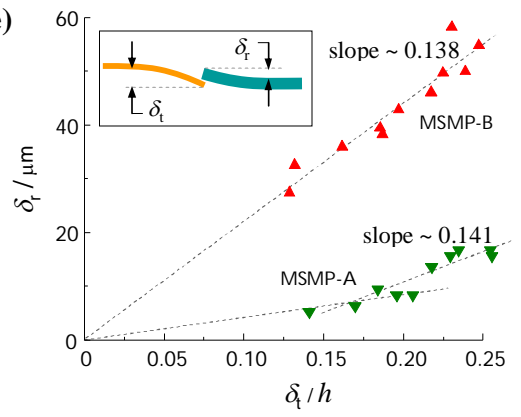

Figure 2. Optical micrographs of (a) a $1134 \mu$ m-high PDMS micropillar integrated with a $50 \mu \mathrm{m}$ PMMA microsphere. Its aspect-ratio is 84. (Scale bar: $100 \mu \mathrm{m}$ ), (b) a $4 \times 4$ array comprising $877.2 \mu \mathrm{m}$-high micropillars integrated with $58 \mu \mathrm{m}$ Ag-coated hollow glass microspheres. (Scale bar: $700 \mu \mathrm{m})$, and (c) the micropillar with the highest aspect-ratio of $112\left(d_{t o p}=9 \mu \mathrm{m}, d_{\text {mid }}=\right.$ $14 \mu \mathrm{m}, d_{\text {base }}=41 \mu \mathrm{m}$, and $h=2.4 \mathrm{~mm}$, scale bar: $\left.100 \mu \mathrm{m}\right)$. (d) The average diameter $d_{\text {ave }}$ of the micropillar plotted as a function of $h$. The dotted lines mark the aspect-ratios. (e) The results of the reference-cantilever method-based measurements. The inset outlines the setup. The dotted lines represent the results of linear fit with zero or non-zero $x$-intersection assumed.

\section{BIO-INSPIRED ACOUSTIC SENSING}

To demonstrate acoustic sensing capabilities of the PDMS MSMPs, we turned MSMP-A in Table 1 into an airflow sensor by adding the optical read-out setup as shown in Figure 3a. There, the PDMS micropillar functions as a flexible waveguide which deforms under airflows. The probe light enters the micropillar from the base and then gets reflected at the top facet back into the photodetector. The flow-induced bending of the micropillar reduces the reflection power through spoiled waveguiding and beam deflection. Critical to the success of the scheme is guaranteeing high-level reflection at the micropillar's top facet. We accomplish it by capping the micropillar with a Ag-coated microsphere and used its surface as the reflector. Figure 3b, $\mathrm{c}$ shows the MSMP-A's deformation in the absence and 
presence of airflow, respectively. In the absence of an air-flow, the MSMP stays straight, producing maximal reflection. Upon contact with a constant airflow, it bends due to the fluidic drag force, which decreases the reflection.

(a)

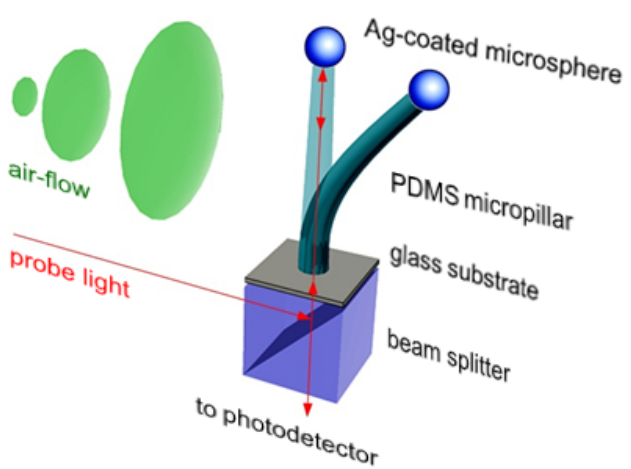

(b)
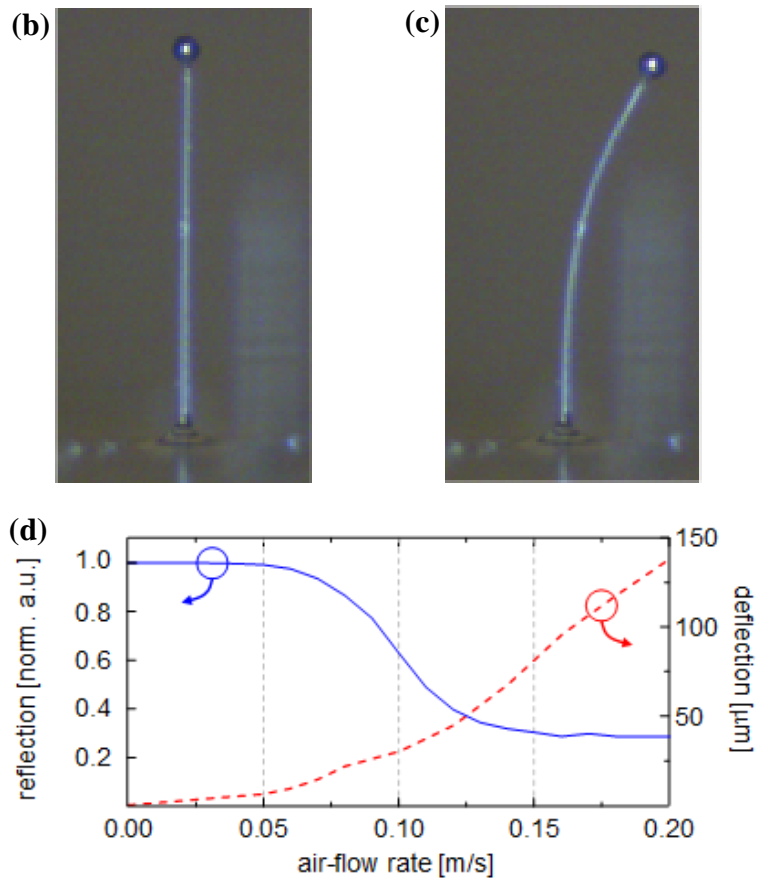

Figure 3. (a) A schematic diagram of an air-flow transducer configuration based on the MSMP (b) and (c) MSMP-A's deformation in response to the absence and presence of an air-flow, respectively. (d) The change in the reflectance as functions of the flow-rate. The deflection length was also plotted for convenience.

The change in the reflection levels is plotted in Figure $3 \mathrm{~d}$ for MSMP-A as a function of the airflow rate. It exhibits a quasi-linear regime sandwiched between two slowly varying curved regimes. The onset of the first curved regime can be defined as the threshold velocity of the MSMP-based air-flow sensors. Figure 3d indicates that it was $0.07 \mathrm{~m} / \mathrm{sec}$ for MSMP A. The curve also indicates that the decrease in the reflection saturates beyond a certain velocity, forming a flat pedestal at $29 \%$ level. It is due mainly to the residual reflection at the air-glass interface, which is not affected by the airflow, and can be reduced with anti-reflection coating. In the quasi-linear regime, the optically retrieved reflection level can function as a good measure of the air-flow velocity. The data represent 10-measurement averages.

\section{CONCLUSION}

In conclusion, we demonstrated an optically interrogated air-flow sensor based on the PDMS micropillars with high aspect ratios ranging from 40 and 112 . Due to the high aspect-ratio and the excellent flexibility of PDMS, the PDMS micropillars deform substantially under airflows, bringing in high sensitivity to small form-factors. The high aspect-ratio PDMS micropillars were fabricated by a new soft-lithography technique based on the combined utility of the in situ thermal hardening and the direct-drawing technique. Our scheme also allows self-aligned capping of the micropillar with microspheres. For this work, we exploited the feature as a way to realize highly reflecting top facets, so that we can interrogate the micropillars' deformation all-optically.

\section{REFERENCES}

[1] Sameoto, D. and Menon, C. "A low-cost, high-yield fabrication method for producing optimized biomimetic dry adhesives", J. Micromech. Microeng. 19, 115002 (2009).

[2] Courbin, L., Denieul, E., Dressaire, E., Roper, M., Ajdari, A., and Stone, H. A. "Imbibition by polygonal spreading on microdecorated surfaces", Nature Materials 6, 661-664 (2007).

[3] Tan, J. L., Tien, J., Pirone, D. M., Gray, D. S., Bhadriraju, K., and Chen, C. S. "Cells lying on a bed of microneedles: An approach to isolate mechanical force", Proc. Natl. Acad. Sci. USA 100, 1484-1489 (2003).

[4] Große, S., Schröder, W., and Brücker, C. "Nano-newton drag sensor based on flexible micro-pillars", Meas. Sci. Technol. 17, 2689-2697 (2006).

[5] Taylor, R. E., Kim, K., Sun, N., Park, S. J., Sim, J. Y., Fajardo, G., Bernstein, D., Wu, J. C., and Pruitt, B. L. "Sacrificial layer technique for axial force post assay of immature cardiomyocytes", Biomed Microdevices 15, 171-181 (2013).

[6] Cheng, Q., Sun, Z., Meininger, G. A., and Almasri, "M. Note: Mechanical study of micromachined polydimethylsiloxane elastic microposts", Rev. Sci. Instrum. 81, 106104 (2010).

[7] Roca-Cusachs, P., Rico, F., Martínez, E., Toset, J., Farré, R., and Navajas, D. "Stability of Microfabricated High Aspect Ratio Structures in Poly(dimethylsiloxane)", Langmuir 21, 5542-5548 (2005)

[8] Paek, J. and Kim, J., "Microsphere-assisted fabrication of high aspect-ratio elastomeric micropillars and waveguides", Nature Communications, 5, 3324 (2014).

[9] Nain, A. S., Wong, J. C., Amon, C., and Sitti, M. "Drawing suspended polymer micro-nanofibers using glass micropipettes", Appl. Phys. Lett. 89, 183105 (2006).

[10] Harfenist, S. A., Cambron, S. D., Nelson, E. W., Berry, S. M., Isham, A. W., Crain, M. M., Walsh, K. M., Keynton, R. S., and Cohn, R. W. "Direct Drawing of Suspended Filamentary Micro- and Nanostructures from Liquid Polymers", Nano Lett. 4, 1931-1937 (2004).

[11] Xing, X., Wang, Y., and Li, B. "Nanofibers drawing and nanodevices assembly in poly(trimethylene terephthalate)", Opt. Express 16, 10815-10822 (2008).

[12] Spina, G. L., Stefanini, C., Menciassi, A., and Dario, P. “A novel technological process for fabricating micro-tips for biomimetic adhesion", J. Micromech. Microeng. 15, 1576-1587 (2005) 
[13] Lee, J., Paek, J., and Kim, J. "Sucrose-based fabrication of 3D-networked, cylindrical microfluidic channels for rapid prototyping of lab-on-a-chip and vaso-mimetic devices", $L a b$ Chip 12, 2638-2642 (2012).

[14] Lebel, L. L., Aissa, B., Khakani, M. A. E., and Therriault, D. "Ultraviolet-Assisted Direct-Write Fabrication of Carbon Nanotube/Polymer Nanocomposite Microcoils", Adv. Mater. 22, 592-596 (2010).

[15] Lee, K., Lee, H. C., Lee, D.-S., and Jung, H. "Drawing Lithography: Three-Dimensional Fabrication of an Ultrahigh-Aspect-Ratio Microneedle", Adv. Mater. 22, 483-486 (2010).

[16] Prakash, M. and Bush, J. W. M. "Interfacial propulsion by directional adhesion", Int. J. Non-Linear Mech. 46, 607-615 (2011).

[17] Gates, R. S. and Pratt, J. R. "Accurate and precise calibration of AFM cantilever spring constants using laser Doppler vibrometry", Nanotechnology 23, 375702 (2012).

[18] Sasoglu, F. M., Bohl, A. J., and Layton, B. E. "Design and microfabrication of a high-aspect-ratio PDMS microbeam array for parallel nanonewton force measurement and protein printing”, J. Micromech. Microeng. 17, 623-632 (2007). 